Jiang, X., et al. Anomalous Nuclear Phenomena Assocoated with Ultrafast Processes. in 7th International

Workshop on Anomalies in Hydrogen / Deuterium loaded Metals. 2006. Asti, Italy: iscmns.org.

\title{
Anomalous Nuclear Phenomena Assocoated with Ultrafast Processes
}

\author{
Xingliu Jiang ${ }^{\mathrm{a}}$, Xiaoping Zhou ${ }^{\mathrm{a}}$, Chun Liu ${ }^{\mathrm{a}}$, Liying Wang ${ }^{\mathrm{a}}$ and Zhongliang Zhang ${ }^{\mathrm{b}}$ \\ a Department of Physics, Beihang University, Beijing 100083, China \\ ${ }^{\mathrm{b}}$ Institute of Chemistry, Chinese Academy of Science, P.O. Box 2709, Beijing 100080, China
}

Quantum physics predicts the existence of an underlying sea of zero-point energy at every point in the universe. If the zero-point energy is real, there is the possibility that it can be tapped as a source of power or be harnassed to generate a propulsive force for space travel.

In our previous papers, anomalous excess heat and localized nuclear reactions on the surface of electrodes in electrolysis cells have been observed. A physical model of transient vortex dynamics with torsion coherence with the zero point energy has been proposed by Xingliu Jiang based on the ultrafast processes of triple phases area of tip effect on the electrode surface. Considering the large equiverlent capacitance of electrochemical double layer, it is presumed that the double layer can exhibit nonlinear electrical response with spatial and temporal variations confined to microscopic areas by tip effect.

Experimental results of transient processes with ultrafast phenomena with nanosecond duration in electrical discharge systems including electrolysis cells have been presented.

\section{Introduction}

When Pons and Fleischmann announced their revolutionary results, the most of scientists in variety of fields rushed to confirm them. No heavy energetic products, like protons tritons or neutrons, and also no gamma rays have been found, they were surprised the negative results. Almost all of these scientists conclude that the idea of "cold Fusion" was a "phony results". However, during the period April 1989 based on tip effect of electrodes, Jiang Xingliu's group has evidently registered the really nuclear products with anomalous energy range by using CR-39 solid detectors, and the phenomena of nuclear transmutations[1].

Since 1989, many laboratories in the world have observed nuclear reactions, excess heat and heat after death in electrochemical systems. T.Ohmori et al found that there is no significant difference in amounts of excess energies between $\mathrm{H}_{2} \mathrm{O}$ and $\mathrm{D}_{2} \mathrm{O}$ electrolysis system for the plasma electrolysis [2]. A.G.Lipson et al found that during the electrolysis of thin $\mathrm{Pd}$ film cathode on the dielectric substrates as well as Pd-black, the alphaparticles ranging from 11.0-16.0 MeV and proton near $1.7 \mathrm{MeV}$ are emitted [3]. The transmuta- tion of Sr, Cs into Mo, Pr with 8 mass number and 4 atomic number increases was observed by Y.Iwamura [4]. Nuclear products with high concentration and tracks with highly collimated lines of low energy nuclear reactions in the electrochemical systems were recorded by CR-39 solid detectors and films at our laboratory $[5,6]$.

It is supposed to use the concept of torsion field to interpret the observed phenomena, typically, the properties of axial acceleration, memory effect, and the polarized nuclear reactions with torsion effect $[7,8]$. The vortex is the assigned workhorse of the universe, which bridges the invisible dark energy and the visible matter.

\section{Vortex dynamics}

The vortex and spiral structures are the archetype that appears at all levels of nature and laboratories, for example, spin of fundamental particles, atom structure with nuclei and orbiting electrons, vortex lattice in superconductors, superfluid, dense plasma focus, lightning, quasar etc. Transient processes produce non-equilibrium system, therefore, create torsion field with high vorticity. Femtosecond laser pulsed excitation of atomic noble gas cluster produce forward ion 
kinetic energies with three orders of magnitude higher than expected. The tip effect of the cathodes in the electrolysis cell appears to enhance those factors to create one nonlinear system with far from the equilibrium condition.

Recent report of dark matter annihilation at the galactic center describes that the cold dark matter near the galactic center is accreted by the central black hole into a dense spike [9]. Particle dark matter annihilation makes the spike to be a compact source of photons, electrons, positrons, protons, antiprotons, and neutrinos. It reminds us for that there is a similarity among pitting corrosion with electrochemical noise, laboratory plasma pinching (dense plasma focus, for example), fast laser induced ion beams [10], and quasar spiral model with high-energy cosmic rays in spiral center in spite of large dimension difference. Comparing the experimental results of electrochemical cells with excess heat and nuclear transmutation to astrophysics phenomena, it is supposed that the investigation of vortex dynamics of torsion coherence with the zero-point energy is essential for tapping the zero-point energy.

Based on observation in the experiments, the following features are considered in order to understand the experimental results with electrical discharge systems.

The local enhancement of the electric field on the cathode surface with the double layer is related to the protrusions and cracks similar to the tip discharge in air or in a vacuum. The current distribution depends strongly on the surface roughness and the work function of the electron emission. A high transient current density $\left(10^{8} \mathrm{~A} / \mathrm{cm}^{2}\right)$ could be expected due to enhanced field and instantaneous process.

\section{Torsion field and tapping the zero point energy}

Transient processes in an open dynamic system always create vortex effect. In metal deuterides, there exist a possible transient dynamic process, which may generate close pairs and clusters of deuteron in the motion to induce a strong screen effect on the repulsive barrier. There is a variety of nonlinear processes which could be taken into consideration. Temperature rises locally. High persistent field in addition to the transient electric and magnetic fields lead to the polarization and coherence effects of the deuterons with quadrupole momentum. Influence of magnetic field on the distribution of deuterium. Azimuth magnetic field created by transient emitted electron current with high flux density due to tip effect causes redistribution of deuteron density on the interface zone of palladium cathode. Experiments showed that the phenomena of the current filaments and the chaotic processes in lattice are extremely sensitive to the magnetic field.

Our current understanding of science is like a puzzle with a large missing piece-zero point energy. Various scientists around the world have been discovering anomalies of phenomena attributed to the conversion of the zero point energy. The combination of ion vortices or vortex rings with abruptly pulsed bucking magnetic fields could result in the direct manifestation of energy and gravitational anomalous. In order to theoretically allow the coherence of the zeropoint energy, two fields of modern physics must combine: theories of the zero point energy interacting with matter, and theories of system selforganization. The facts of production of nuclear reactions in room temperature solid-state setting, including 'cold' and 'bubble' fusion, and ultrafast laser-plasma interaction could be explained by physical model of transient vortex dynamics with torsion coherence with the zero point energy[11].

\section{Experimental results}

Ultrafast processes have been observed in the electrolysis system under certain conditions.

In previous works, excess heat in open $\mathrm{Pd} / \mathrm{D}_{2} \mathrm{O}$ electrochemical system by using a Calvet calorimeter[12] has been reported. The Calvet calorimeter is one of standard equipments in thermokinetic experiments.

Fig.1 shows that the pulsed current waveforms corresponding pulsed voltage waveforms have been recorded for DC voltage electrolysis cell by computer multichannel registrated system under certain conditions. 


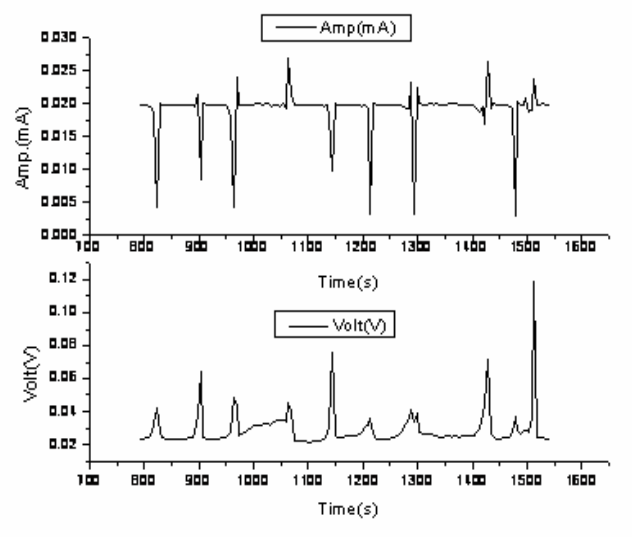

Figure 1. Electrolysis current and voltage pulses by computer multichannel registrated system

In our plasma electrolysis experiments, the cell was a 700 cc Pyrex glass vessel which contained 500 cc of $0.2 \mathrm{M} \mathrm{K}_{2} \mathrm{CO}_{3}$ electrolyte.If the anode was a piece of metallic wire netting and the cathode was tungsten rod, the width of the waveforms was less than $50 \mathrm{~ns}$ (fig 2)when the voltage was up to 150 volt, however, if the cathode was a piece of wire netting and anode was tungsten rod, at the same voltage, the width of the waveforms could reach to 300 microsecond.

\section{Conclusion}

The phenomena of so-called cold fusion with excess heat and anomalous nuclear reactions could be explained by transient processes in the triple phase area of tip effect. Vortex dynamics of nonequilibrium system with nonlinear process leads to tapping the zero point energy.

\section{REFERENCES}

1. Jiang Xingliu, Lei Jinzhi, Torsion field and tapping the zero-point energy in an electrochemical system, J. of New Energy, 4(2), 93(1999)

2. T. Ohmori, H. Yamada, S. Narita and T.

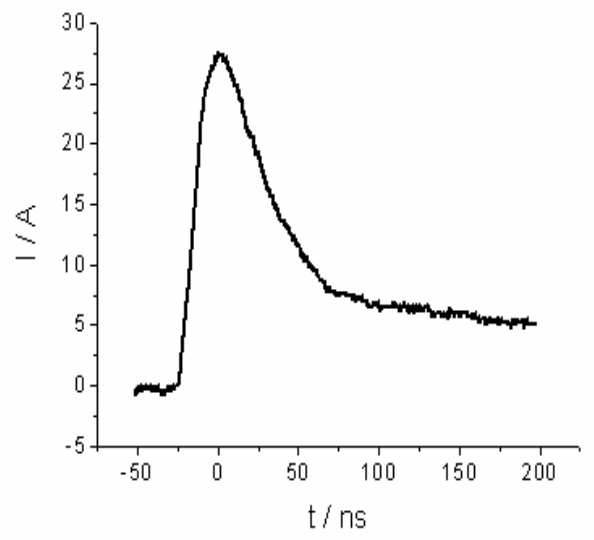

Figure 2. The pulsed current waveform of plasma electrolysis with $\mathrm{K}_{2} \mathrm{CO}_{3}$ electrolyte

Mizuno, Proceedings of ICCF-9, Beijing, China, p.284 (2002)

3. A.G. Lipson, A.S. Roussetski, G.H. Miley, C.H. Casatno, Proceedings of ICCF-9, Beijing, China, p.218 (2002)

4. Y. Iwamura, N. Gotoh, T. Itoh and I. Toyoda, Fusion Technol. 33, 476(1998)

5. Xing-liu Jiang, Jin-zhi Lei, Li-jun Han, Dynamic Casimir Effect in an Electrochemical System, J. New Energy, Vol.3, No. 4, 47(1999)

6. Xing-liu Jiang, Chang-ye Chen, Li-jun Han, Tip Effect and Nuclear Active Sites, Proc. of the 7th Intern Conf. on Cold Fusion, Vancouver, April 1998, pp.175

7. Don. Read, Excitation and Extraction of Vacuum Energy via EM-Torsion Field Coupling Theoretical Model. J. New Energy, Vol. 3, No. 2/3, p.130 (1998)

8. Xing-zhong Li, Proceedings of ICCF-9, Beijing, China, p.1 (2002)

9. P. Gondolo, J. Silk, Dark matter annihilation at galactic center, Phys. Rev. Lett. 83, 1719 (1999)

10. BPS, Laser light in stream of protons out, Physics Today, Jan.2000, p.9

11. B. Naranjo, J.K. Gimzewski and S. Putterman, Observation of nuclear fusion driven 
by a pyroelectric crystal, Nature, 434, 1115(2005)

12. Wu-Shou Zhang, Zhao-Fu Zhang, ZhongLiang Zhang, Primary Calorimetric results on Closed Pd/D2O Electrolysis Systems By Calvet Calorimetry, Proceedings of The 9th International Conference on Cold Fusion, Tsinghua University, Beijing, China, p431 